\title{
Fatal Acute Diclofenac-Induced Rhabdomyolysis in A Pediatric Patient
}

\author{
Ahmet Güzel, Betül Orhaner Biner, Serap Karasalihoğlu, Hakan Aylanç \\ Department of Pediatrics, Faculty of Medicine, Trakya University, Edirne, Turkey
}

\begin{abstract}
Diclofenac, a widely used nonsteroidal anti-inflammatory drug, has been reported to cause fatal drug-induced rhabdomyolysis very rarely . We report a case of a 13-month-old girl who developed fatal rhabdomyolysis after administration of intramuscular diclofenac for relief of pain caused by a scalding burn injury. Rhabdomyolysis due to diclofenac has not been reported in children. Here, we present the first case report to demonstrate rhabdomylysis secondary to diclofenac administration.
\end{abstract}

Key Words: Rhabdomyolysis, diclofenac, child, drug, side effect

Received: 17.02.2009 Accepted: 30.03.2009

\section{Introduction}

Fatal drug-induced rhabdomyolysis is a very rarely encountered complication of Diclofenac (DCF), a widely used nonsteroidal anti-inflammatory drug. All reported cases of rhabdomyolysis complicating DCF overdose appeared in adults following intentional overingestion $(1,2)$.

We report a case of a 13-month-old girl treated with intramuscular DCF that was complicated by rhabdomyolysis.

\section{Case}

This case report describes a 13 month old patient weighing $10 \mathrm{~kg}$, who was transferred to our facility after sustaining a scald injury. The spilling of scalding water caused burn injury. She had 10\% deep dermal ("second-degree") burns on her anterior thorax. At the state hospital where she was initially evaluated, she received a single $40 \mathrm{mg}$ intramuscular injection of DCF for pain control.

Fifteen minutes after admission, she suffered cardiorespiratory collapse. The patient was then intubated, resuscitated and transferred to our pediatric emergency department on the $4^{\text {th }}$ hour. The child was not taking any other medications. On arrival, her vital signs included a heart rate of 182 beats per minute, a blood pressure of $90 / 50 \mathrm{~mm} \mathrm{Hg}$, oxygen saturation $94 \%$ by positive pressure ventilation, and a temperature of $37.5^{\circ} \mathrm{C}$. There was no evidence of musculoskeletal trauma.

Her initial laboratory analysis is presented in Table 1. This is notable for an elevated LDH, elevated liver functions tests, creatinine kinase of $12.926 \mathrm{IU} / \mathrm{L}$, and prolonged prothrombin time (PT). Arterial blood gas analysis revealed metabolic acidosis (pH 6.98, pO2 $50.4 \mathrm{mmHg}, \mathrm{pCO} 244.0 \mathrm{mmHg}, \mathrm{HCO} 3$ $8.6 \mathrm{mmol} / \mathrm{l}, \mathrm{BE}:-27.3)$. Her urine was dark red in colour and urinalysis showed a specific gravity of $1.020,1+$ protein, trace ketones, and 4+ blood; in the microscopic examination, 8
RBCs/high power field (HPF), 5 WBCs/HPF, no bacteria, and no casts were seen. Urine myoglobin was not avaliable.

She was continued on mechanical ventilation with midazolam for sedation. She received aggressive intravenous fluid resuscitation with sodium bicarbonate to maintain urine output of $3 \mathrm{~mL} / \mathrm{kg} / \mathrm{h}$. Her serum CK peaked at $35.905 \mathrm{IU} / \mathrm{L}$ on the second day then normalized by day 5 . With aggressive IV fluids the patient's, BUN and creatinine levels returned to normal by day 4 . The patient received four units of fresh frozen plasma. Although only midazolam infusion was used as a sedative for ventilator treatment, chest wall rigidity developed, and despite using high ventilatory pressures of up to 50-60 cm, no chest movement could be achieved. As a result, pneumothorax and pneumoperitoneum developed and the patient died on the sixth day of hospitalization.

\section{Discussion}

Diclofenac is a commonly used drug, but there are very few reports about fatal drug-induced rhabdomyolysis due to its misuse. Diclofenac has been associated with rhabdomyolysis in three described cases in the literature and acute renal failure occurred in all of these patients $(1,2)$. To our knowledge, this is the first reported pediatric case of DCF-induced rhabdomyolysis.

As the pharmacokinetics of DCF has not been investigated in pediatric patients, it is not recommended for children. Safe dosage has not been established, especially for parenteral usage in children. It is not licensed in children under 1 year, and carries a higher risk of gastrointestinal and other various serious side effects. The usual dose is $0.5-1 \mathrm{mg} / \mathrm{kg}$ with oral and rectal doses being equivalent (3).

Rhabdomyolysis is a syndrome resulting from the lysis of muscle cells and the leakage of intracellular contents into the 
Table 1. Laboratory Analysis of the Patient

\begin{tabular}{|c|c|c|c|c|}
\hline & Initial & 2. day & 3. day & 4. day \\
\hline $\mathrm{Hb}(\mathrm{g} / \mathrm{dl})$ & 11.2 & 9.8 & 10.8 & 10.4 \\
\hline WBC (x109/L) & 20.000 & 6.700 & 18.100 & 15.200 \\
\hline PLT (x10\%/L) & 354.000 & 198.000 & 129.000 & 430.000 \\
\hline BUN (7-40mg/dl) & 36 & $72-93$ & 49 & 38 \\
\hline Creatinine $(0.6-1.1 \mathrm{mg} / \mathrm{dl})$ & 0.8 & $0.8-0.9$ & 0.6 & 0.6 \\
\hline Uric acid (2.6-7.2mg/dl) & 6.4 & $12.9-10.5$ & 9.7 & 2.9 \\
\hline LDH (0-450IU/L) & 4256 & $5.759-8.350$ & 5328 & 4290 \\
\hline ALT (<40IU/L) & 123 & $1.213-895$ & 393 & 456 \\
\hline AST $(<50$ IU/L) & 56 & $752-706$ & 600 & 66 \\
\hline CK (40-226IU/L) & 12926 & $25.642-35.905$ & 10.293 & 481 \\
\hline $\mathrm{Ca}^{+2}(8.4-10.2 \mathrm{mg} / \mathrm{dl})$ & 8.7 & 6.7-7.0 & 7.7 & 9.3 \\
\hline Albumin $(3.5-5 \mathrm{~g} / \mathrm{dl})$ & 3.5 & $3.7-3.5$ & 3.5 & 4.2 \\
\hline PT/INR (11-15 sec/1-1.5) & $23.1 / 2.0$ & 19.0/1.6 & $16.1 / 1.3$ & 13.1/1.02 \\
\hline
\end{tabular}

plasma and the extracellular fluid (4). These contents include myoglobin, creatine phosphokinase, aldolase, lactate dehydrogenase, serum glutamic-oxaloacetic transaminase, and potasium (5). The most common causes of rhabdomyolysis are quite diverse, including direct muscle injury (crush, burns, electrical shock, freezing, prolonged immobility), muscle overuse (excessive exercise, seizures), ischemia, viral infections, metabolic disorders (hypophosphatemia, hypokalemia), genetic defects, inflammatory myopathies, toxins, and drugs $(4,6)$. Although our patient had burns, the cause of rhabdomyolysis was thought to be DCF because in burns, deeper tissue damage leads to rhabdomyolysis.

Drug-induced rhabdomyolysis is a serious, sometimes life-threatening and there are several case reports in the literature involving some common drugs that caused rhabdomyolysis $(1,2,7,8)$. Antihistamines, anesthetics, and amphetamines have recently been implicated as the etiology of drug-induced rhabdomyolysis in several pediatric patients $(1,2,7,8)$. Hanna et al reported a child who developed severe metabolic acidosis, progressive hypoxia, and rhabdomyolysis during a maintenance infusion of propofol for the treatment of refractory status epilepticus (9). The occurrence of rhabdomyolysis after ingestion of an overdose of isoniazid in a 17-year old girl who recovered completely has also been reported (10). Nearly all reported cases concerning drug-induced rhabdomyolysis are associated with unintentional overdose in children or intentional overdose in adults. In our case, the rhabdomyolysis resulted from unintentional overdose with DCF (4 mg/kg IM).

The presenting clinical characteristics of rhabdomyolysis are skeletal muscle injury, pigmented urine, and some aspect of renal dysfunction. The complications of rhabdomyolysis include acute renal failure, metabolic instability (hyperkalemia, hyperphosphatemia, hyperuricemia, hypocalcemia), disseminated intravascular coagulation, compartment syndrome, and peripheral neuropathy (5). The treatment of drug-induced rhabdomyolysis consists of elimination of exposure, sedation, external cooling, hydration, alkalinization, and correction of electrolyte abnormalities (6).

In conclusion, the patient described shows the importance of strict adherence to recommended dosages of drugs especially in children, and emphasized the need for increased awareness of DCF-induced rhabdomyolysis.

\section{Conflict of Interest}

No conflict of interest was declared by the authors.

\section{References}

1. Knobloch K, Rossner D, Gössling T, Lichtenberg A, Richter M, Krettek C. Rhabdomyolysis after administration of diclofenac. Unfallchirurg 2005;108:415-7.

2. Delrio FG, Park Y, Herzlich B, Grob D. Case report: diclofenac-induced rhabdomyolysis. Am J Med Sci 1996;312:95-7. [CrossRef]

3. British National Formulary for children. London, UK: BMJ Publishing Group, 2006.

4. Coco JT, Klasner AE. Drug-induced rhabdomyolysis. Current Opinion in Pediatrics 2004;16:206-10. [CrossRef]

5. Counselman FL, Rhabdomyolysis. Emergency Medicine Comprehensive study guide. Tintinalli JE, Kelen GD, Stapczynski JS Eds. Fifth edition. McGraw-Hill Companies. 2000;271:1841-5.

6. Larbi EB. Drug-induced rhabdomyolysis. Ann Saudi Med. 1998;18:525-30.

7. Selimoglu O, Basaran M, Ugurlucan M, Ogus T. Rhabdomyolysis Following Accidental Intraarterial Injection of Local Anesthetic. Angiology 2009;60:120-1.

8. Stucka KR, Mycyk MB, Leikin JB, Pallasch EM. Rhabdomyolysis associated with unintentional antihistamine overdose in a child. Pediatr Emerg Care. 2003;19:25-6. [CrossRef]

9. Hanna JP, Ramundo ML Rhabdomyolysis and hypoxia associated with prolonged propofol infusion in children. Neurology. 1998;50:301-3.

10. Blowey $\mathrm{DL}$, Johnson $\mathrm{D}$, Verjee Z. Isoniazid-associated rhabdomyolysis. Am J Emerg Med. 1995;13:543-4. [CrossRef] 